\title{
LOW ENERGY ION-MOLECULE REACTIONS
}

\author{
AND \\ CHEMIIONIZATION KINETICS
}

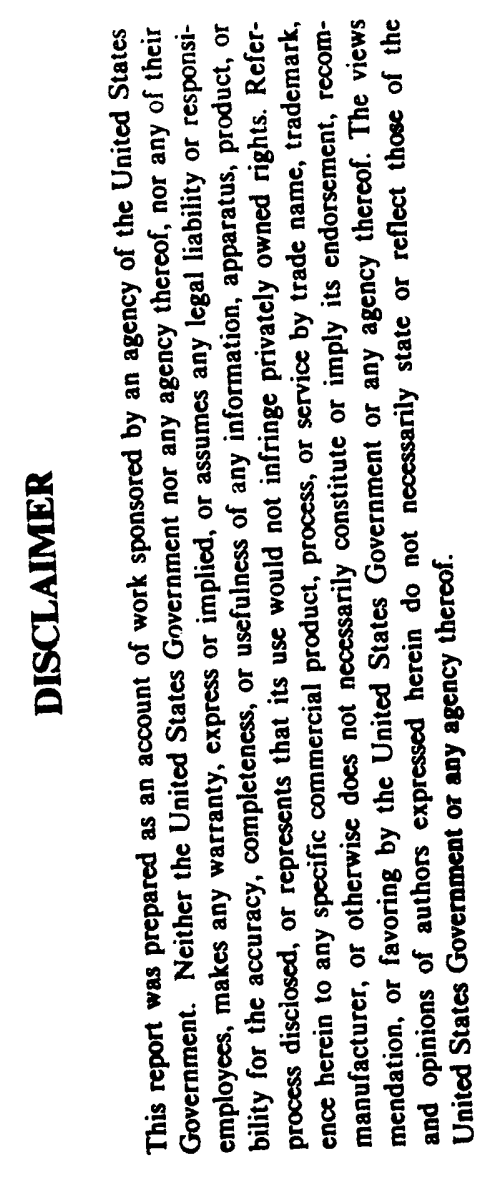

\author{
Progress Report \\ James M. Farrar \\ University of Rochester \\ Rochester, New York 14627
}

February 1, 1992 - January 31, 1993

Prepared for the U. S. Department of Energy under Grant No. DE-FG02-88ER13849

Date submitted: September 24, 1992

\section{MASTER}




\section{NOTICE}

This report was prepared as an account of work sponsored by the United States Government. Neither the United States nor the Department of Energy, nor any of their employees, nor any of their contractors, subcontractors, or their employees, makes any warranty, express or implied, or assumes any legal liability or responsibility for the accuracy, completeness, or usefulness of any information, apparatus, product or process disclosed or represents that its own use would not infringe privately-owned rights. 
Table of Contents

Research Overview

Negative Ion Reaction Dynamics

Statement of Unexpended Funds 


\section{Research Overview}

Our research program is devoted to understanding the dynamics of elementary ionic collisions at the level of the underlying potential surface by measuring energy and angular distributions of reactively scattered products with crossed beam methods over the relative center-of-mass energy range from 0.3 to several $\mathrm{eV}$. During the past few year, we have emphasized reaction dynamics of anionic species important in combustion, with special emphasis on $\mathrm{O}^{-}$in proton and hydrogen atom transfer reactions with $\mathrm{NH}_{3}$, $\mathrm{H}_{2} \mathrm{O}, \mathrm{HF}$, and $\mathrm{CH}_{4}$.

\section{Negative Ion Reaction Dynamics}

During the period of February 1, 1992 until the present, we have devoted our efforts into the reaction dynamics of $\mathrm{O}$ with $\mathrm{H}_{2} \mathrm{O}, \mathrm{NH}_{3}$, and $\mathrm{HF}$. We bave published two papers on the first two of these systems, and have acquired initial results on the reaction of $\mathrm{O}+\mathrm{HF}$.

We have studied reactive and nonreactive collisions between $\mathrm{O}^{-}$and $\mathrm{H}_{2} \mathrm{O}$ over the collision energy range from 0.67 to $1.07 \mathrm{eV}$. Kinetic energy analysis of the $\mathrm{O}$ scattered nonreactively from $\mathrm{H}_{2} \mathrm{O}$ shows two components: the first arises from direct scattering and is nearly quasielastic, while the second occurs at significantly lower barycentric energies and corresponds to $\mathrm{O}$ ejected without reaction from electrostatically bound $\mathrm{O} \cdot \mathrm{H}_{2} \mathrm{O}$ complexes formed by approaching reagents. This latter flux is significantly more intense than the reactive $\mathrm{OH}^{-}$flux. The kinetic energy distributions for the low energy $\mathrm{O}$ nonreactive flux are in qualitative agreement with 
statistical phase space theory, although recoil distributions that model the exit channel by an $r^{-4}$ potential underestimate the kinetic energy release. The reactive flux distributions show a strong energy dependence. At the lowest collision energy, the $\mathrm{OH}^{-}$ is produced through two pathways, the first involving the participation of a complex living a fraction of a rotational period, the second producing $\mathrm{OH}^{-}$strongly backward scattered and with a much broader kinetic energy distribution. With increasing collision energy, the complex contribution to the scattering falls off rapidly, and product formation moves from the backward hemisphere to the forward direction. The angular distribution asymmetries at the lowest collision energies can be interpreted in terms of the osculating model for chemical reactions taking place in a fraction of a rotational period of the intermediate complex. This model suggests that the complex lifetime is $\sim 250 \mathrm{fs}$ at collision energies between 0.7 and $0.8 \mathrm{eV}$, a result in good agreement with RRKM calculations. The kinetic energy distributions at these energies are in good agreement with statistical phase space theory calculations. At the highest collision energies, still below the threshold for impulsive stripping collisions, the $\mathrm{OH}^{\cdot}$ product is scattered sharply forward with a broad kinetic energy distribution peaking near $0.3 \mathrm{eV}$. We interpret the high energy dynamics as direct, but still involving significant interaction among all four atoms. The rapid variation in dynamics over a narrow collision energy range is attributed to the heavy-light-heavy mass combination of this system.

We have also studied reactive and nonreactive collisions of $\mathrm{O}$ with $\mathrm{NH}_{3}$ at relative collision energies of 0.65 and $1.24 \mathrm{eV}$. We observed a significant contribution to the collision dynamics from nonreactive encounters between the reagents. In 
addition to elastic scattering, we observed a direct contribution to this nonreactive scattering with a very strong angular dependence of energy transfer on scattering angle. A third contribution to nonreactive scattering arose from $\mathrm{O} \cdot \mathrm{NH}_{3}$ collision complexes that regenerate the reactants. In these collisions, approximately $80 \%$ of the incident translational energy is transformed into vibrational-rotational excitation of the $\mathrm{NH}_{3}$ reagent. The kinetic energy distribution is in reasonable agreement with statistical phase space theory calculations. We also observed reactive collisions. The hydrogen atom transfer process to yield $\mathrm{OH}^{-}$is exothermic by $0.11 \mathrm{eV}$ and exhibits direct dynamics at all collision energies. Proton transfer to form $\mathrm{NH}_{2}^{-}$, endothermic by $0.9 \mathrm{eV}$, was studied as its deuterium analog and was observed only at the higher collision energy, and took place with very small cross section. The product kinetic energy distributions for the hydrogen atom transfer reaction approach a Gaussian form at the higher collision energy, and we ascribe that behavior to the impulsive nature of reactive collisions in which the ground state vibrational wavefunction of the $\mathrm{N}-\mathrm{H}$ bond to be broken is reflected onto product translational energy states through the "corner" of the potential energy surface. Such a Franck-Condon picture of the reaction is a consequence of the highly skewed potential energy surface associated with the heavylight-heavy mass combination.

The results from the $\mathrm{O}^{-}+\mathrm{HF}$ reaction have given us unexpected insight (and pleasure!) into the dynamics of this simple proton transfer reaction. The reaction is exothermic by $0.48 \mathrm{eV}$, such that at collision energies near $0.4 \mathrm{eV}$, the lower limit of our crossed beam instrument, we can produce $O H$ in the vibrational states $v^{\prime}=0,1$, or 2 . 
Our first data on this reaction demonstrated very clear structured kinetic energy distributions with features clearly assignable to each of the product vibrational states of OH. Furthermore, the product angular distributions show very clear bimodal forwardbackward character, consistent with the observation of a transient $\mathrm{O} \cdot \mathrm{HF}$ complex. This is an important observation: a wide variety of proton transfer reactions have been observed through spectroscopic observations of the products, either through infrared emission from vibrationally excited products, or by laser-induced fluorescence. In many of these studies, the ionic systems, in which a deep well is assumed to exist owing to the participation of a transient electrostatic bound complex, have been contrasted with the corresponding neutrals, where such a well does not exist. As an example, Leone and co-workers ${ }^{1}$ have contrasted the ionic reaction

$$
\mathrm{Cl}^{-}+\mathrm{HBr} \rightarrow \mathrm{HCl}+\mathrm{Br}
$$

with the neutral system

$$
\mathrm{Cl}+\mathrm{HBr} \rightarrow \mathrm{HCl}+\mathrm{Br} \text {. }
$$

Their comment on this comparison is that the product state distributions for both reactions are quite comparable, although only in the ionic case is a well thought to be involved in the dynamics. The value of our crossed beam experiments is the fact that the angular distributions for the $\mathrm{O}^{-}+\mathrm{HF}$ have demonstrated unambiguously that a complex governs the dynamics. Further, our measurements have also confirmed an observation noted in Leone's work that increasing translational energy channels energy preferential into product vibration, rather than translation, as is the usual dogma of Heavy + Heavy-Light collision systems. It certainly appears that the well is responsible 
for this behavior. The combination of the spectroscopic studies of Leone with our crossed beam measurements makes the $\mathrm{O}^{-}+$HF system one of the most thoroughly studied anion-neutral systems. We stress that the $A+B C$ system is one of the best candidates for "translational spectroscopy" of the reaction products, but angular momentum partitioning must cooperate in order for such measurements to be successful. We are very pleased that this system has been so favorable, and thus so informative. We look forward to ab initio studies of the surface, as well as dynamical studies on that surface. We hope our studies stimulate such work.

Our work for the third year of this grant will continue with studies of hydrogen atom and proton transfer reactions of $\mathrm{O}^{-}$and $\mathrm{OH}^{-}$with $\mathrm{CH}_{4}$, other hydrocarbons, and hydrogen halides. The latter systems will extend our results on product state-resolved dynamical studies. Because we measure both reactive and non-reactive fluxes, our data are also good candidates for studies of inversion procedures, ${ }^{2}$ in which deflection functions and reaction opacities can be extracted. These studies are underway, and we expect publishable results shortly.

\section{References}

1. K. Knutsen and S. R. Leone, J. Chem. Phys., 96, 298 (1992).

2. M. A. Collins and R. G. Gilbert, Chem. Phys. Lett., 41, 108 (1976); S. M. McPhail and R. G. Gilbert, Chem. Phys., 34, 319 (1978). 
Publications, February 1, 1992 - January 31, 1993

J. M. Farrar, "Crossed Beam Studies of Ion-Molecule Complexes: Collisions and Clusters", in Dynamics of Ion-Molecule Complexes, edited by W.L. Hase, JAI Press, 1992, p. 0000.

D. F. Varley, D. J. Levandier, and J. M. Farrar, "Dynamics of the Reaction of Owith $\mathrm{H}_{2} \mathrm{O}$ : Reactive and Nonreactive Decay of Collision Complexes," J. Chem. Phys. 96, 8806 (1992).

D. J. Levandier, D. F. Varley, and J.M. Farrar, "Low Energy Ion-Molecule Reaction Dynamics: Complex and Direct Collisionis of $\mathrm{O}^{-}$with $\mathrm{NH}_{3}$, " J. Chem. Phys. 97, 4008 (1992). 
Statement of Unexpended Funds

No unexpended funds are anticipated for the grant, "Low Energy Ion-Molecule Reactions and Chemiionization Kinetics", DE-FG02-88ER13849, for the period February 1, 1992 through January 31, 1993. 

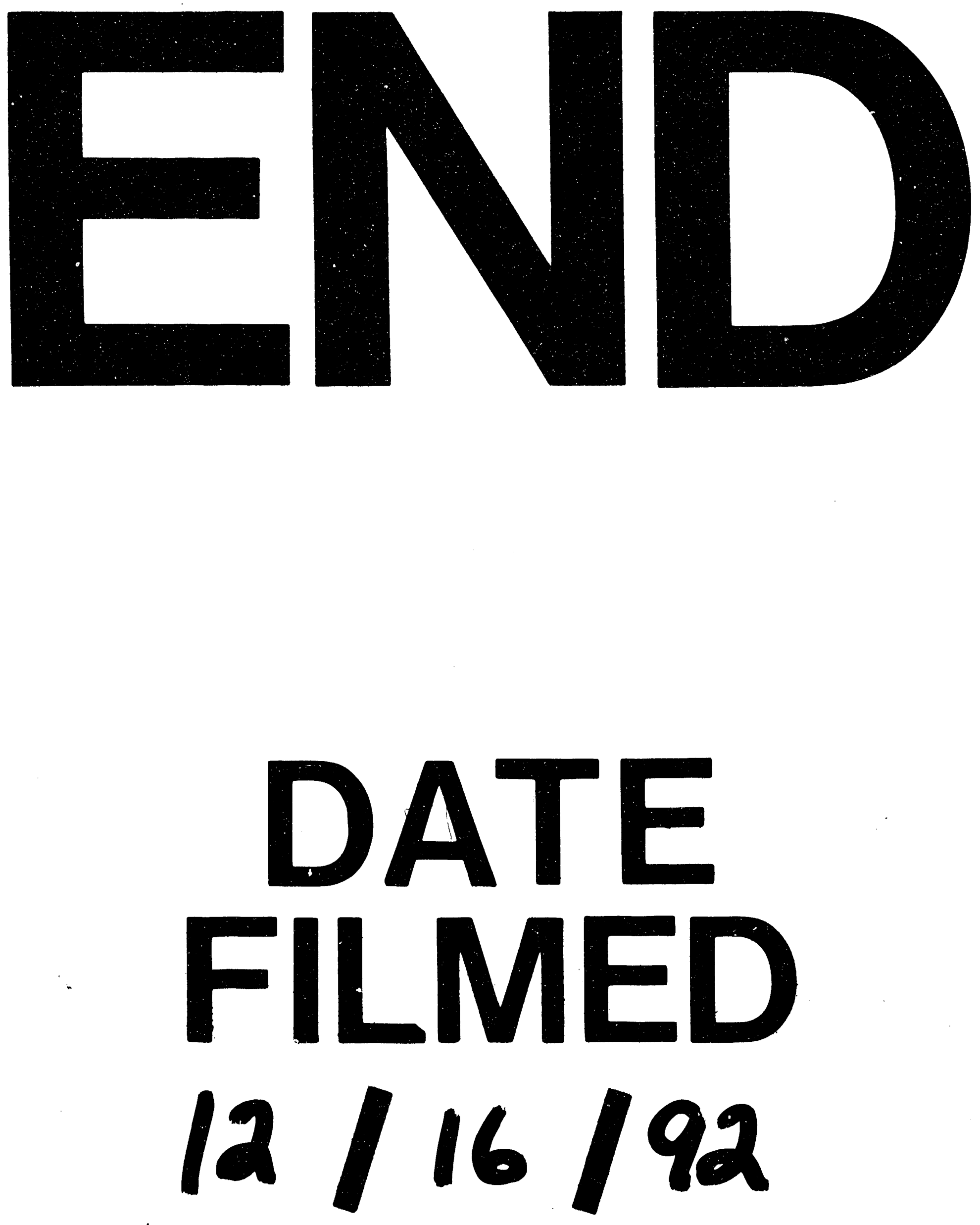
